\title{
Potencial de ADSORÇÃo do NANOCOMPÓsito híbrido de GRAFENO MAGNÉTICO PARA REMOÇÃO DE HERBICIDA DIURON
}

\author{
Ana Carolynna Severo de Almeida ${ }^{1}\left[\right.$, Lara de Sousa Soletti $^{2}{ }^{\circledR}$, Andressa Jenifer Rubio ${ }^{3}$,

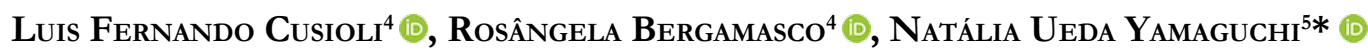

1 Graduação em Engenharia Ambiental e Sanitária, Centro Universitário de Maringá - Unicesumar, Av. Guedner, 1610, bloco 07, CEP 87050-900, Maringá, PR, Brasil.

2 Graduanda em Engenharia Química, Centro Universitário de Maringá - Unicesumar, Av. Guedner, 1610, bloco 07, CEP 87050-900, Maringá, PR, Brasil.

3 Mestrado em Tecnologias Limpas, Centro Universitário de Maringá - Unicesumar, Av. Guedner, 1610, bloco 07, CEP 87050-900, Maringá, PR, Brasil. 4 Departamento de Engenharia Química, Universidade Estadual de Maringá, Av. Colombo, 5790, bloco D90, CEP 87020-900, Maringá, PR, Brasil.

5 Mestrado em Tecnologias Limpas, Centro Universitário de Maringá - Unicesumar, ICETI, Av. Guedner, 1610, bloco 07, sala 08, CEP 87050-900, Maringá, PR, Brasil.

* Autor para correspondência: natalia.yamaguchi@unicesumar.edu.br

Recebido em 29 de dezembro de 2019. Aceito em 06 de julho de 2020. Publicado em 31 de julho de 2020.

Resumo - Dentre os processos para remover contaminantes da água, a adsorção se distingue como uma tecnologia de remediação superior. O grafeno vem se destacando como material adsorvente devido às suas propriedades únicas. Este estudo foi realizado utilizando um nanocompósito magnético híbrido de grafeno e ferrita de manganês (NCGM) para o tratamento de água contaminada com herbicida diuron, desenvolvendo possíveis processos alternativos de tratamento de água e efluentes. O potencial de adsorção do adsorvente foi avaliado em ensaios batelada. Foram realizados estudos cinéticos, sendo as isotermas de adsorção também estudadas. Os resultados mostraram que o NCGM apresentou uma capacidade máxima de adsorção $7,9 \mathrm{mg} \mathrm{g}^{-1}$ para a temperatura de $45 \stackrel{\circ}{\circ} \mathrm{C}$ após $4 \mathrm{~h}$ de tempo de contato. Os dados obtidos na cinética de adsorção ajustaram-se ao modelo de pseudo-segunda ordem, indicando um processo químico envolvido. O modelo de Freundlich foi melhor para o ajuste dos dados obtidos para as isotermas de adsorção, indicando um processo de adsorção heterogêneo e multicamadas. O processo se mostrou endotérmico, sendo favorecido pelo aumento de temperatura. Portanto, concluiu-se que o NCGM se apresentou como um potencial material adsorvente para tratamento de águas contaminadas com diuron.

Palavras-chave: Adsorvente; Ferrita de Manganês; Óxido de Grafeno; Tratamento de Água.

ADSORPTION POTENTIAL OF THE HYBRID NANOCOMPOSITE OF MAGNETIC GRAPHENE FOR DIURON HERBICIDE REMOVAL

Abstract - Among the processes used to remove contaminants from water, adsorption is distinguished as a superior remediation technology. Graphene has stood out as an adsorbent material due to its unique properties. This study was carried out using a hybrid magnetic nanocomposite of graphene and manganese ferrite (NCGM) for treating water contaminated with diuron herbicide, developing possible alternative water and effluent treatment processes. The adsorption potential of the adsorbent was evaluated in batch scale tests. Kinetic studies were performed and the adsorption isotherms were also studied. The results showed that NCGM presented a maximum adsorption capacity of $7.9 \mathrm{mg} \mathrm{g}^{-1}$ at $45^{\circ} \mathrm{C}$ after $4 \mathrm{~h}$ of contact time. The dada obtained in adsorption kinetics adjusted to the pseudo-second order model, indicating a chemical process involved. Freundlich's model presented better adjustment for the data obtained in the adsorption isotherms, indicating a heterogeneous and multilayer adsorption process. The process proved to be endothermic, being favored by temperature increase. Therefore, it was concluded that NCGM presented itself as a potential adsorbent material for treatment of diuron contaminated waters. 
KEYwORDs: Adsorbent; Manganese Ferrite; Graphene Oxide; Water Treatment.

Potencial DE ADSORCIÓN DEL NANOCOMPUESTO HÍBRIDO DE GRAFENO MAGNÉTICO PARA ELIMINAR EL HERBICIDA DIURON

RESumen - Entre los procesos para eliminar contaminantes del agua, la adsorción se distingue como una tecnología de remediación superior. El grafeno se ha destacado como material adsorbente debido a sus propiedades únicas. Se utilizó en este estudio un nanocompuesto magnético híbrido de grafeno y ferrita de manganeso (NCGM) para el tratamiento de agua contaminada con herbicida diuron, desarrollando posibles procesos alternativos de tratamiento de aguas y efluentes. El potencial de adsorción del adsorbente se evaluó en ensayos por lotes. Se realizaron estudios cinéticos y también se estudiaron las isotermas de adsorción. Los resultados mostraron que el NCGM presentó una capacidad de adsorción máxima de $7.9 \mathrm{mg} \mathrm{g}^{-1}$ a $45{ }^{\circ} \mathrm{C}$ después de $4 \mathrm{~h}$ de tiempo de contacto. Los resultados de la cinética de adsorción se ajustan al modelo de pseudo-segundo orden, lo que indica un proceso químico involucrado. El modelo de Freundlich fue mejor para ajustar los resultados para las isotermas de adsorción, lo que indica un proceso de adsorción heterogéneo y multicapa. El proceso resultó ser endotérmico, favorecido por el aumento de temperatura. Por lo tanto, se concluyó que NCGM se presentaba como un material adsorbente potencial para el tratamiento de aguas contaminadas con diuron.

Palabras Clave: Adsorbente; Ferrita de Manganeso; Óxido de Grafeno; Tratamiento de Aguas.

\section{INTRODUÇÃO}

Agroquímicos representam os produtos mais amplamente encontrados em corpos d'água superficiais e subterrâneos em todo o mundo, devido à ampla utilização em áreas agrícolas e urbanas. Isso acontece, pois apenas menos de $10 \%$ dos pesticidas pulverizados atingiram seus objetivos, enquanto outros pesticidas persistem no solo e atingem os corpos de água por escoamento agrícola, causando a extinção de espécies e os sintomas de intoxicação (Rawtani et al. 2018). Os agroquímicos compreendem uma variedade de moléculas com propriedades distintas que conferem diferentes graus de persistência ambiental, mobilidade e potenciais tóxicos, carcinogênicos, mutagênicos e teratogênicos, ou algum efeito endócrino a vários organismos não-alvo, incluindo seres humanos (Armas et al. 2007).

Devido ao considerável aumento de relatórios envolvendo agrotóxicos no meio aquoso, surge a necessidade de criar sistemas de tratamento mais eficazes e acessíveis para removê-los do meio ambiente; visto que, a maioria apresenta alta estabilidade química e baixa biodegradabilidade tornando-se difíceis de remover (Oller et al. 2006).

O diuron (3- (3,4-diclorofenil)-1,1-dimetilureia) é um herbicida comumente usado em áreas agrícolas e não agrícolas. É quimicamente estável, persistente no ambiente, carcinogênico e genotóxico (Bumroongsakulsawat et al. 2020). A molécula de diuron pode ser encontrada no solo, nas águas superficiais e subterrâneas, sendo levemente tóxico para mamíferos e aves, além de moderadamente tóxico para invertebrados aquáticos (Zbair et al. 2020). O diuron tem se tornado gradualmente um contaminante emergente responsável pela redução da qualidade da água. Assim, existe uma necessidade de aplicar técnicas adequadas para reduzir a lixiviação de herbicidas dos locais de aplicação, a fim de minimizar o seu impacto nas águas superficiais e/ou subterrâneas (Al-Shaalan et al. 2019).

Várias técnicas têm sido desenvolvidas para remoção de agroquímicos. Dentre os vários processos para remover contaminantes da água, a adsorção é uma tecnologia de remediação superior, em termos de baixos custos dos adsorventes, flexibilidade e simplicidade de design, de fácil operação e alta eficiência (Ghaedi et al. 2014). No entanto, resposta lenta, menos especificidade e sensibilidade são algumas das desvantagens de tais técnicas. Nos últimos tempos, a nanotecnologia surgiu como uma ferramenta de ajuda para a detecção e remediação de agroquímicos (Rawtani et al. 2018). 
Materiais à base de carbono têm sido explorados extensivamente para aplicações de adsorção devido à sua boa estabilidade, diversidade estrutural, baixa densidade, e aplicabilidade para produção em larga escala (Chowdhury e Balasubramanian 2014). Tendo em vista a grande variedade de nanomateriais para a adsorção, o grafeno tem atraído grande atenção da comunidade científica, devido às suas surpreendentes propriedades mecânicas, elétricas, térmicas, ópticas e elevada área específica (Guan et al. 2020; Martín e Escarpa 2014). Embora o grafeno e o óxido de grafeno sejam bons adsorventes para muitos poluentes, a sua eficiente remoção da água após o processo de tratamento continua a ser um desafio (Kumar et al. 2014; Neolaka et al. 2020; Pu et al. 2018).

Para superar este problema de separação, uma tecnologia inovadora que tem ganhado muita atenção é a utilização de materiais magnéticos para um processo de separação mais fácil. A separação magnética pode ser feita usando campos magnéticos para a sua separação de fases de soluções aquosas, proporcionando um método atrativo e eficaz em termos de custos e praticidade de operação (Wang et al. 2011). Muitas pesquisas vêm sendo realizadas para integrar o grafeno com nanopartículas magnéticas, uma vez que estes compósitos híbridos são prováveis de apresentarem também funcionalidades melhoradas em relação à adsorção de contaminantes (Lingamdinne et al. 2019; Ruiz et al. 2019; Tasmia et al. 2020; Zhang et al. 2011).

Desta forma, o presente trabalho teve como objetivo a utilização do nanocompósito híbrido de grafeno magnético (NCGM) para o tratamento de água avaliando o seu potencial de adsorção do herbicida diuron em soluções aquosas.

\section{Material e Métodos}

\section{Sintese de óxido de grafeno}

A rota mais comum para obter quantidades representativas de óxido de grafeno inicia-se pela oxidação do grafite para óxido de grafite (Martín e Escarpa 2014; Singh et al. 2011). Os primeiros procedimentos para a síntese de óxido de grafite foi desenvolvida há muitas décadas por Brodie (1859) e posteriormente por Staudenmaier (1898)\{Staudenmaier, 1898 \#81@@author-year;Staudenmaier, 1898 \#81\}. Tanto o método de Standenmaier quanto o método de Brodie geram gás $\mathrm{ClO}_{2}$, que devem ser manuseados com precaução pois apresentam alta toxicidade e tendência a decomposição em ar podendo produzir explosões, além de possuírem tempo de reação que podem levar dias.

Hummers e Offeman (1958) desenvolveram uma metodologia diferente para produção de óxido de grafeno, com tempo de reação relativamente menor e ausência de gás $\mathrm{ClO}_{2}$. O método de Hummers é a técnica mais utilizada para sintetizar óxido de grafeno até os dias atuais, com pequenas modificações. Portanto, este método foi selecionado para produzir óxido de grafeno (Hummers e Offeman 1958; Ji et al. 2013; Kovtyukhova et al. 1999; Yamaguchi et al. 2016). Primeiramente foi realizada uma pré-oxidação do grafite, utilizando $5 \mathrm{~g}$ de grafite em pó, 2,5 g de $\mathrm{K}_{2} \mathrm{~S}_{2} \mathrm{O}_{8}$ e 2,5 g de $\mathrm{P}_{2} \mathrm{O}_{5}$ em $18 \mathrm{~mL}$ de $\mathrm{H}_{2} \mathrm{SO}_{4} 98 \%$, mantidos à $80 \stackrel{\circ}{\circ} \mathrm{C}$ durante $5 \mathrm{~h}$ sob agitação constante, em seguida foi diluído em $1 \mathrm{~L}$ de água deionizada, lavado com água deionizada, coletado por filtração, e seco em estufa à $60^{\circ} \mathrm{C}$ por $12 \mathrm{~h}$. Um grama de grafite pré-oxidado, $23 \mathrm{~mL}$ de ácido sulfúrico $98 \%$, sob agitação e $3 \mathrm{~g}$ de $\mathrm{KMnO}_{4}$ foram adicionados lentamente. Manteve-se à temperatura de $35{ }^{\circ} \mathrm{C}$ sob agitação durante 2 horas, e em banho de gelo, adicionou-se lentamente $46 \mathrm{~mL}$ de água deionizada, mantendo-se a temperatura inferior a $50{ }^{\circ} \mathrm{C}$, agitando-se a amostra por mais $2 \mathrm{~h}$. Para finalizar a reação adicionou-se $140 \mathrm{~mL}$ de água deionizada e 2,5 $\mathrm{mL}$ de peróxido de hidrogênio $30 \%$ e lavou-se a mistura com $250 \mathrm{~mL}$ de solução aquosa de $\mathrm{HCl} 10 \%$. A mistura foi centrifugada e lavada com água deionizada três vezes a $3700 \mathrm{rpm}$ por $15 \mathrm{~min}$, removendo-se o sobrenadante. Finalmente, secou-se em estufa a $60^{\circ} \mathrm{C}$ por $12 \mathrm{~h}$ (Wang et al. 2011; Yamaguchi et al. 2016). 
O NCGM foi sintetizado com base em metodologias solvotérmicas de uma única etapa encontradas na literatura (Cai et al. 2014; Chella et al. 2015; Yamaguchi et al. 2016). Tipicamente, $100 \mathrm{mg}$ de óxido de grafeno, $1 \mathrm{~g}$ de cloreto de ferro $\left(\mathrm{FeCl}_{3} \cdot 6 \mathrm{H}_{2} \mathrm{O}\right)$ e $0,376 \mathrm{~g}$ de cloreto de manganês $\left(\mathrm{MnCl}_{2} \cdot 4 \mathrm{H}_{2} \mathrm{O}\right)$ foram dispersos em $30 \mathrm{~mL}$ de etileno glicol por meio de ultrasonicação durante $3 \mathrm{~h}$. Em seguida, $3 \mathrm{~g}$ de acetato de sódio foram adicionados e dissolvidos por meio de agitação magnética durante $30 \mathrm{~min}$. A mistura foi transferida para uma autoclave de aço inox com cápsula interna em teflon a $200{ }^{\circ} \mathrm{C}$ por $10 \mathrm{~h}$. O material resultante foi lavado várias vezes com etanol e seco em forno a $60{ }^{\circ} \mathrm{C}$ por $12 \mathrm{~h}$.

\section{Experimentos de adsorção}

Os experimentos de adsorção foram realizados em batelada utilizando frascos de $250 \mathrm{~mL}$ de polipropileno, com volume de $25 \mathrm{~mL}$ de solução de herbicida diuron $5 \mathrm{mg} \mathrm{L}^{-1}$ em incubadora shaker de bancada Lucadema modelo Luca-223 com controle de temperatura. As amostras foram coletadas e separadas magneticamente, filtradas, e a concentração foi determinada em espectrofotômetro HACH DR5000 em comprimento de onda de $247 \mathrm{~nm}$. Todos os ensaios foram realizados em duplicatas. A capacidade de adsorção foi determinada de acordo com a Equação (1).

$$
q=\frac{C_{i}-C_{f}}{m} \times V
$$

Onde q é a capacidade de adsorção, $C_{i}$ é a concentração inicial e $C_{f}$ é a concentração final do herbicida diuron em $\mathrm{mg} \mathrm{L}^{-1}$, $\mathrm{M}$ é a massa do adsorvente em gramas e $\mathrm{V}$ é o volume da solução em litros.

Foram realizados testes preliminares de $4 \mathrm{~h}$ à temperatura de $25{ }^{\circ} \mathrm{C}$ para avaliar os efeitos provocados por alterações no pH (pH 7 e pH 10), na massa de adsorvente (0,025 e 0,050 g) e velocidade de agitação das amostras (100 e $150 \mathrm{rpm})$. As condições não alteradas foram a agitação de $150 \mathrm{rpm}$ para os ensaios que não avaliavam a velocidade de agitação, 0,025 g de NCGM para os ensaios sem avaliação da alteração da massa de adsorvente, e pH 7 para ensaios que não estavam avaliando a variação do pH na adsorção.

A cinética de adsorção foi determinada por ensaios realizados nas condições ótimas encontradas nos ensaios preliminares, sendo 0,025 g de NGCM, $150 \mathrm{rpm}, \mathrm{pH}$ 7, e o volume $25 \mathrm{~mL}$ e temperatura $25{ }^{\circ} \mathrm{C}$. Neste ensaio as amostras foram coletadas em intervalos de tempo predeterminados (15 min, 1, 2, 3, 5, 8 e $18 \mathrm{~h}$ ) e em duplicatas.

Os resultados da cinética de adsorção foram analisados de acordo com os modelos cinéticos principais, o modelo de pseudo primeira-ordem e o pseudo-segunda ordem, sendo representados pelas Equações (2) e (3), respectivamente.

$$
\begin{aligned}
& \ln \left(\mathrm{q}_{\mathrm{e}}-\mathrm{q}_{\mathrm{t}}\right)=\ln \left(\mathrm{q}_{\mathrm{e}}\right)-\mathrm{k}_{1} t \\
& \frac{1}{\mathrm{q}_{\mathrm{t}}}=\frac{1}{\mathrm{k}_{2} \mathrm{q}_{\mathrm{e}}^{2}}+\frac{1}{\mathrm{q}_{\mathrm{e}}}
\end{aligned}
$$

onde $\mathrm{q}_{\mathrm{e}}$ e $\mathrm{q}_{\mathrm{t}}$ são as quantidades de soluto adsorvidas no tempo de equilíbrio e no tempo $\mathrm{t}$ em horas, respectivamente, $\mathrm{k}_{1}$ é a constante da taxa de adsorção de pseudo-primeira ordem e $\mathrm{k}_{2}$ é a constante de equilíbrio da taxa de adsorção de pseudo-segunda ordem.

Após a determinação das condições ótimas de adsorção e do tempo de equilíbrio, foram realizados ensaios para determinação da isoterma de adsorção. Sucederam-se três ensaios com diferentes temperaturas, 15,25 e $45^{\circ}$ 
C. Os ensaios foram realizados com as mesmas condições anteriores, 0,025 g de NCGM, volume de solução 25 $\mathrm{mL}, 150 \mathrm{rpm}$, sendo que neste caso as concentrações de herbicida diuron foram de 1, 3, 5, 10 e $20 \mathrm{mg} \mathrm{L}^{-1}$.

Os dados das isotermas de adsorção obtidos foram ajustados aos modelos de Langmuir e Freundlich para obter mais informações sobre o tipo de adsorção, utilizando-se as Equações (4) e (5) para cada modelo, respectivamente (Freundlich 1906; Langmuir 1918).

$$
\begin{aligned}
\mathrm{q}_{\mathrm{e}} & =\frac{\mathrm{q}_{\max } \mathrm{K}_{\mathrm{L}} \mathrm{C}_{\mathrm{e}}}{1+\mathrm{K}_{\mathrm{L}} \mathrm{C}_{\mathrm{e}}} \\
q_{e} & =\mathrm{K}_{\mathrm{F}} C_{e}^{1 / n}
\end{aligned}
$$

onde $\mathrm{q}_{\mathrm{e}}$ é a quantidade de adsorvente adsorvido por unidade de massa de adsorvente $\left(\mathrm{mg} \mathrm{g}^{-1}\right), \mathrm{C}_{\mathrm{e}}$ é a concentração de adsorvente não adsorvido em solução no equilíbrio $\left(\mathrm{mg} \mathrm{L}^{-1}\right), \mathrm{K}_{\mathrm{L}}=$ Constante de equilíbrio de Langmuir $\left(\mathrm{L} \mathrm{mg}^{-1}\right), \mathrm{K}_{\mathrm{F}}$ a constante de Freundlich $\left(\mathrm{L} \mathrm{mg}^{-1}\right)$ e $\mathrm{n}$ o expoente de Freundlich.

\section{Resultados e Discussão}

\section{Otimizaçãa das condições de adsorção}

Diferentes condições de adsorção foram avaliadas para a determinação das melhores condições de operação.

Por meio dos ensaios de otimização de condições de adsorção observou-se que a remoção por adsorção com a solução em pH 7 foi maior quando comparada com a solução em pH 10 (Figura 1). A carga do NCGM é negativa em $\mathrm{pH} \mathrm{7,} \mathrm{conforme} \mathrm{trabalhos} \mathrm{anteriores,} \mathrm{sendo} \mathrm{o} \mathrm{potencial} \mathrm{zeta} \mathrm{em} \mathrm{torno} \mathrm{de}-17 \mathrm{mV}$ e em $\mathrm{pH} 10$, o potencial zeta se torna ainda mais negativo, próximo de $-28 \mathrm{mV}$ (Yamaguchi et al. 2016). A taxa de hidrólise da molécula do herbicida diuron é insignificante em $\mathrm{pH}$ neutro, mas aumenta à medida que as condições se tornam fortemente ácidas ou alcalinas (Salvestrini et al. 2002), ou seja, é ligeiramente ionizável, seu valor de pKa corresponde a 13,5 e, dessa forma, no valor de $\mathrm{pH} 10$ o diuron é levemente ionizado negativamente em solução, dessa forma acaba gerando uma repulsão eletrostática em $\mathrm{pH}$ elevado, devido a carga negativa do nanocompósito (de Souza e dos Santos 2018).

O experimento inicial utilizando as velocidades da mesa agitadora, $100 \mathrm{rpm}$ e $150 \mathrm{rpm}$ possibilitou concluir que a maior velocidade apresentou melhores resultados (Figura 1B). Isso ocorreu, possivelmente, devido ao maior contato entre o poluente e o adsorvente que o aumento da agitação forneceu.

No ensaio preliminar para verificar a remoção de diuron com a variação da massa de NCGM, ou seja, a massa de adsorvente, observou-se que a remoção do herbicida foi de 68,4\% após 4 h com 0,05 g, de acordo com a Figura 1C, sendo maior que a remoção com metade da massa de adsorvente, $0,025 \mathrm{~g}(49,4 \%)$. Contudo a capacidade de adsorção (q) diminuiu com o aumento da massa, desta forma, para massa de 0,025 $\mathrm{g}$ a capacidade de adsorção foi de $2,95 \mathrm{mg} \mathrm{g}^{-1}$, enquanto que para massa de $0,05 \mathrm{~g}$, a capacidade de adsorção foi de $1,71 \mathrm{mg} \mathrm{g}^{-1}$. A partir dessa análise optou-se por manter a massa de adsorvente em $0,025 \mathrm{~g}$. 
Figura 1. Remoção do herbicida diuron para diferentes condições de A) pH, B) velocidade de agitação e C) massa de nanocompósito híbrido de grafeno magnético (NCGM).

A)

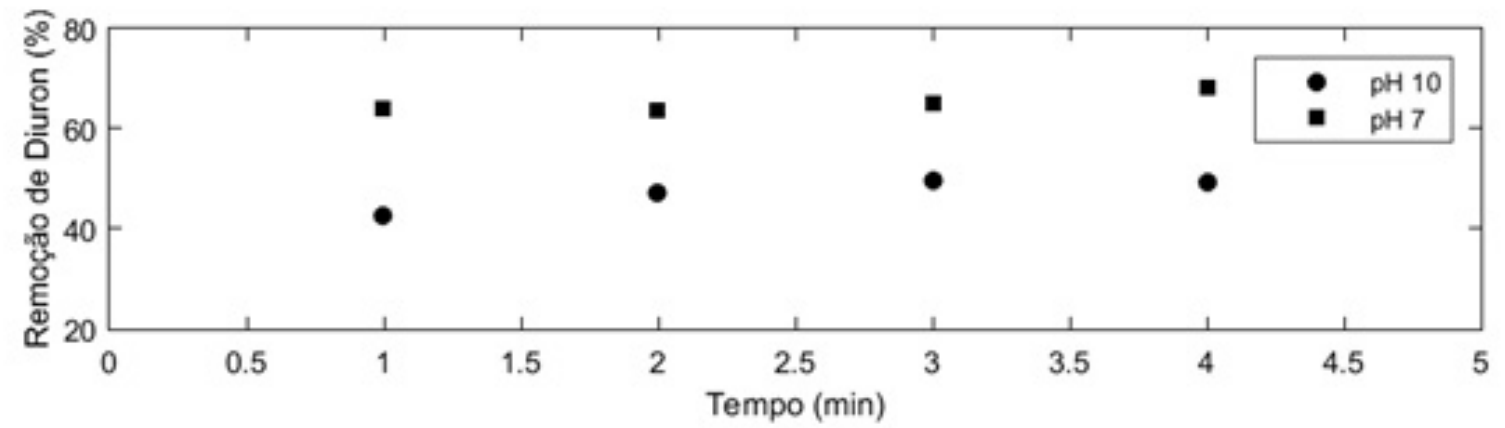

B)

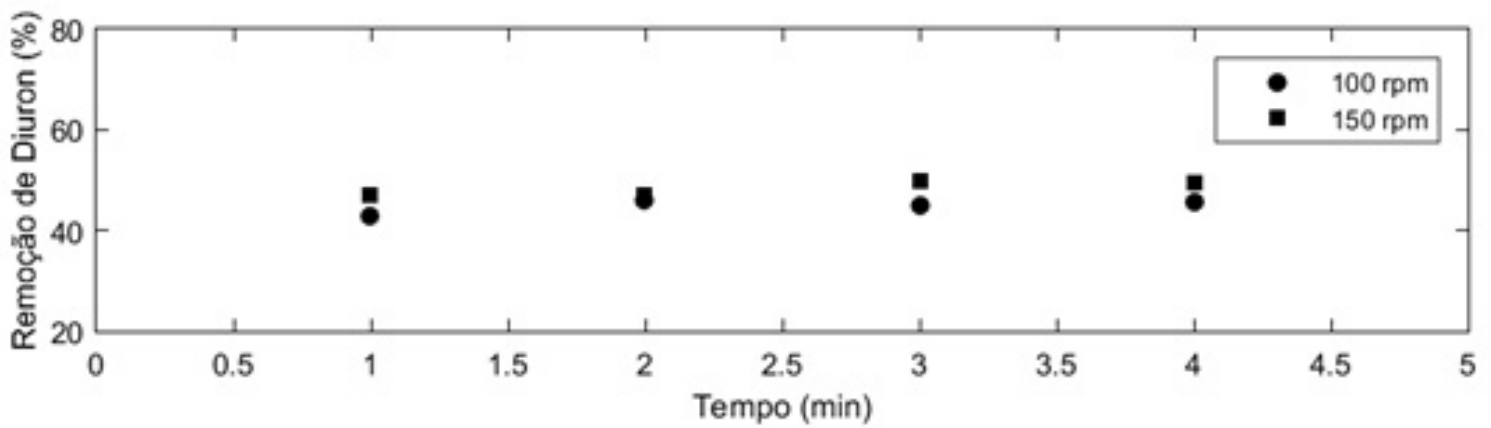

C)

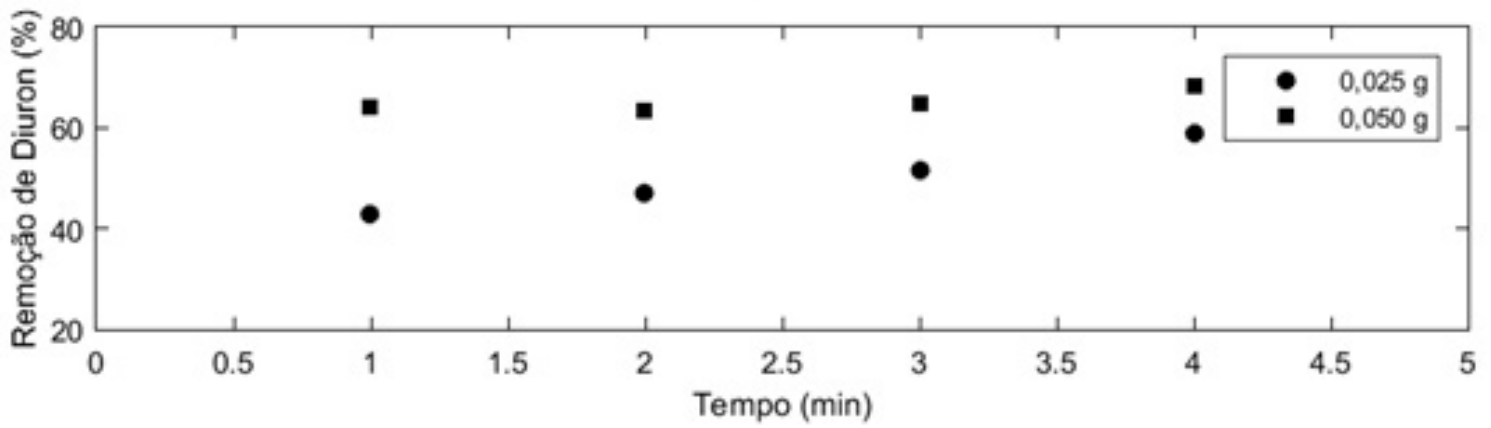

Cinética de adsorção

A adsorção é um processo dependente do tempo, por isso, a predição da etapa determinante da velocidade é importante para a avaliação do adsorvente e o projeto de sistemas de remoção de contaminantes de águas e efluentes.

Foi possível observar que o tempo necessário para atingir o equilíbrio para adsorção do herbicida diuron foi de aproximadamente $8 \mathrm{~h}$ com capacidade de adsorção de $2,65 \mathrm{mg} \mathrm{g}^{-1}$ (Figura 2). Porém, por ser um tempo muito longo, foi determinado um tempo de experimento de $4 \mathrm{~h}$, pois após $3 \mathrm{~h}$ de experimento mais de $90 \%$ da capacidade máxima de adsorção já era atingida. Assim o tempo escolhido para os ensaios de isotermas de adsorção foram de $4 \mathrm{~h}$. 
Figura 2. Cinética de adsorção para o diuron utilizando a massa de nanocompósito híbrido de grafeno magnético (NCGM).

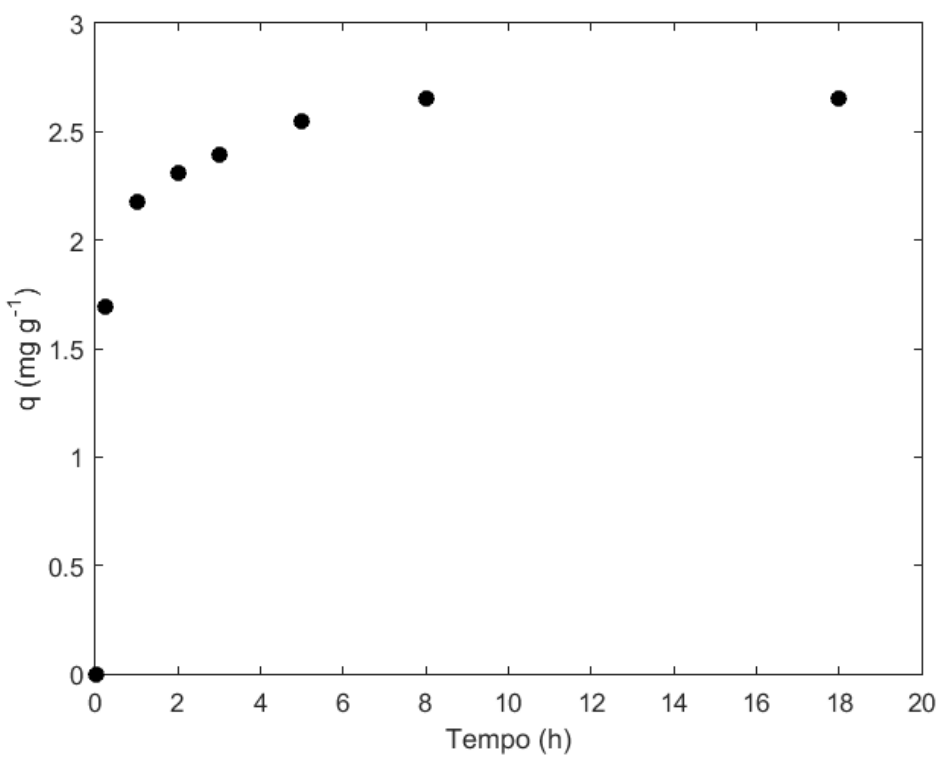

A cinética de adsorção descreve a velocidade no qual as moléculas do adsorvato são adsorvidas pelo adsorvente. Esta velocidade depende das características físico-químicas do adsorvato e do adsorvente e das características da solução. Dentre os principais modelos cinéticos, podemos citar o modelo de pseudo-primeira ordem e o pseudo-segunda-ordem. Estes modelos foram ajustados de acordo com as Equações (2) e (3), os resultados estão apresentados na Figura 3 e Tabela 1.

Figura 3. Ajuste de A) modelo cinético de pseudo-primeira ordem e B) modelo cinético de pseudo-segunda ordem para adsorção de diuron pelo nanocompósito híbrido de grafeno magnético (NCGM).
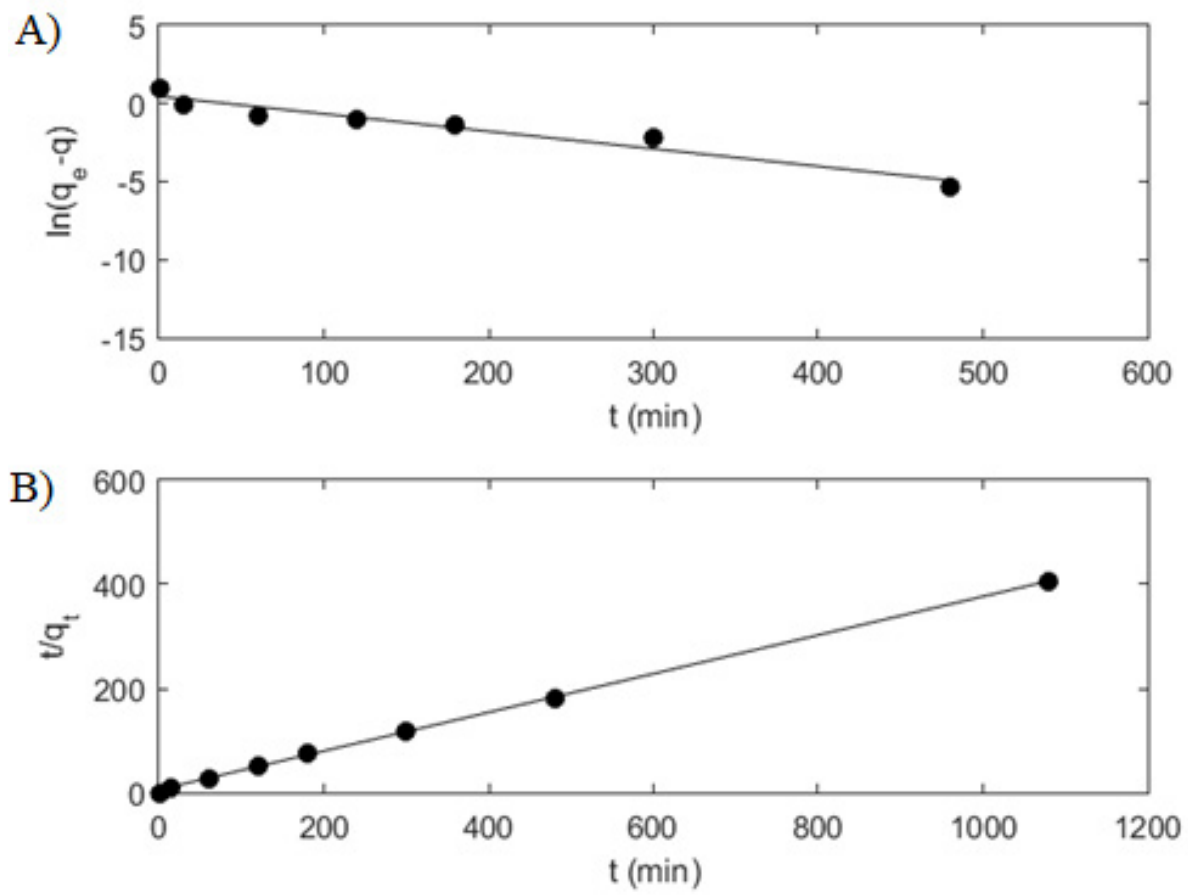
Tabela 1. Parâmetros dos modelos de cinéticos de adsorção.

\begin{tabular}{ccccccc}
\hline Experimental & \multicolumn{3}{c}{ Pseudo-primeira ordem } & \multicolumn{3}{c}{ Pseudo-segunda ordem } \\
$\mathrm{q}_{\mathrm{e}}\left(\mathrm{mg} \mathrm{g}^{-1}\right)$ & $\mathrm{K}_{1}\left(\mathrm{~min}^{-1}\right)$ & $\mathrm{q}_{\mathrm{e}}\left(\mathrm{mg} \mathrm{g}^{-1}\right)$ & $\mathrm{R}^{2}$ & $\mathrm{~K}_{2}\left(\mathrm{~g} \mathrm{mg}^{-1} \mathrm{~min}^{-1}\right)$ & $\mathrm{q}_{\mathrm{e}}\left(\mathrm{mg} \mathrm{g}^{-1}\right)$ & $\mathrm{R}^{2}$ \\
\hline 2,655 & 0,011 & 1,581 & 0,9436 & 0,343 & 2,695 & 0,9998 \\
\hline
\end{tabular}

De acordo com a Figura 3, pode-se observar que os dados obtidos para cinética de adsorção se ajustaram melhor ao modelo de pseudo-segunda ordem. Neste modelo, a reação baseia-se no pressuposto de quimissorção envolvendo forças de valência por meio de partilha ou troca de elétrons entre o adsorvente e o adsorvato, diferente do modelo de pseudo-primeira ordem, em que o modelo assume como sendo uma adsorção molecular de não-dissociação no adsorvente, ou seja, fisissorção (Ruthven 1984).

Baseado nos valores do coeficiente de correlação $\left(R^{2}\right)$ obtidos pelos dados experimentais apresentados na Tabela 1, pode-se confirmar que a equação em que melhor se ajustou a cinética de adsorção foi o de pseudosegunda ordem $\left(\mathrm{R}^{2}=0,9998\right)$, determinando ser o melhor modelo. Isso também é confirmado ao observar o valor de capacidade máxima de adsorção calculado pelo modelo de pseudo-segunda ordem $\left(2,695 \mathrm{mg} \mathrm{g}^{-1}\right)$, sendo muito próximo do valor experimental $\left(2,655 \mathrm{mg} \mathrm{g}^{-1}\right)$.

\section{Isoterma de adsorção}

Os melhores resultados de adsorção foram obtidos à temperatura de $45{ }^{\circ} \mathrm{C}$, sendo a capacidade máxima de adsorção obtida para esta temperatura $7,9 \mathrm{mg} \mathrm{g}^{-1}$ (Figura 4). Este resultado indicou que a temperatura influenciou na adsorção do herbicida, sugerindo possivelmente um processo endotérmico.

Figura 4. Isotermas de adsorção.

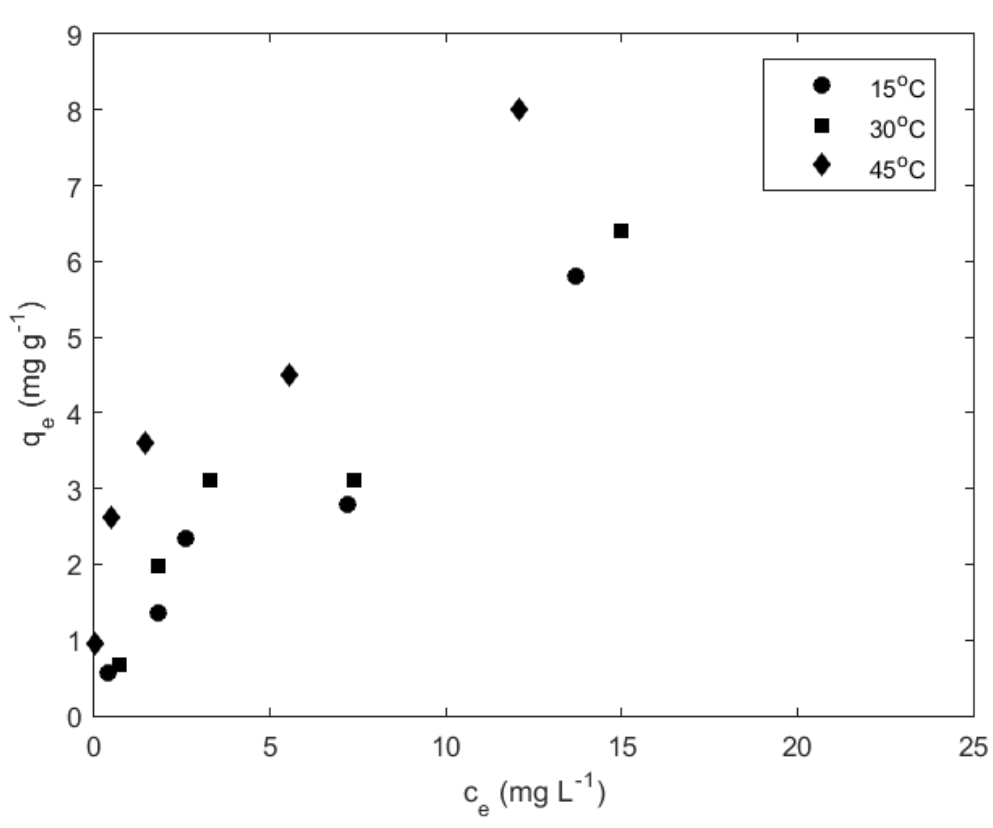

$\mathrm{Na}$ Tabela 2 estão apresentados os valores obtidos para os parâmetros dos ajustes dos modelos de Langmuir e Freundlich. De acordo com os resultados dos ajustes dos modelos de isotermas, notou-se que os dados do processo de adsorção do herbicida diuron pelo NCGM não se ajustaram adequadamente ao modelo de Langmuir, visto que o $\mathrm{R}^{2}$ foi tão próximo de 1 . Este resultado indica também que as constantes $\mathrm{K}_{\mathrm{L}}$ e $\mathrm{q}_{\mathrm{m}}$ não necessariamente 
indicam a energia de ligação entre adsorbato-adsorvente e a quantidade máxima adsorvida. Pode-se observar até mesmo, que neste caso, a constante $\mathrm{q}_{\mathrm{m}}$ calculada foi maior para a temperatura de $15{ }^{\circ} \mathrm{C}$ em comparação com as outras temperaturas, diferentemente do que ocorreu experimentalmente, comprovando que esse modelo não representa adequadamente os dados obtidos (Paz et al. 2018).

Tabela 2. Parâmetros dos modelos de Langmuir e Freundlich.

\begin{tabular}{ccccccc}
\hline \multirow{2}{*}{$\mathrm{T}\left({ }^{\circ} \mathrm{C}\right)$} & \multicolumn{3}{c}{ Modelo de Langmuir } & \multicolumn{3}{c}{ Modelo de Freundlich } \\
& $\mathrm{q}_{\mathrm{m}}\left(\mathrm{mg} \mathrm{g}^{-1}\right)$ & $\mathrm{K}_{\mathrm{L}}\left(\mathrm{L} \mathrm{mg}^{-1}\right)$ & $\mathrm{R}^{2}$ & $\mathrm{~K}_{\mathrm{F}}\left(\mathrm{L} \mathrm{mg}^{-1}\right)$ & $\mathrm{n}$ & $\mathrm{R}^{2}$ \\
\hline 15 & 21,68 & 0,028 & 0,96 & 0,82 & 1,32 & 0,95 \\
30 & 13,41 & 0,038 & 0,99 & 0,63 & 1,32 & 0,97 \\
45 & 7,87 & 0,558 & 0,93 & 2,88 & 2,65 & 0,97 \\
\hline
\end{tabular}

A Tabela 2 apresenta também os resultados dos parâmetros calculados para o modelo de Freundlich. Neste caso, os dados apresentaram uma melhor representatividade dos dados, porém os valores de coeficiente de correlação também não se aproximaram de 1 . Este resultado indica possivelmente que $\mathrm{K}_{\mathrm{F}}$ e $\mathrm{n}$, representam as constantes empíricas indicadoras da capacidade e da intensidade da energia de adsorção. Sugerindo que a adsorção ocorreu em uma superfície heterogênea e amorfa, com diferentes energias de adsorção não restritas à adsorção monocamada ou à capacidade finita de adsorção do adsorvente, ou seja, este modelo sugere uma adsorção de tipo multicamadas comum em materiais heterogêneos (Ruthven 1984).

\section{ConClusões}

Os resultados obtidos dos ensaios preliminares indicaram que o processo de adsorção pode ser otimizado nas condições de $\mathrm{pH} \mathrm{7,} \mathrm{maior} \mathrm{agitação,} \mathrm{e} \mathrm{concentração} \mathrm{de} \mathrm{adsorvente} \mathrm{de} 1 \mathrm{~g} \mathrm{~L}^{-1}$. A análise do modelo cinético, resultou em um melhor ajuste para o modelo de pseudo-segunda ordem indicando um processo de adsorção químico, enquanto que a análise das isotermas de adsorção levaram ao melhor ajuste ao modelo de Freundlich, indicando um processo de adsorção em multicamadas e em superfície heterogênea. Considerando os resultados obtidos, NCGM apresentou um potencial bastante promissor no processo de adsorção do herbicida diuron, especialmente devido à crescente demanda por materiais eficientes e magneticamente separáveis para remediação ambiental. No entanto, algumas questões, como a produção em larga escala de grafeno e nanopartículas, aglomeração de partículas adsorventes, toxicidade dos nanomateriais na água pós-tratada e o custo da produção em massa, são grandes obstáculos para aplicações em larga escala utilizando efluentes reais. Assim, são sugeridos estudos futuros utilizando sistemas piloto e ensaios reais de águas residuais.

\section{Agradecimentos}

Instituto Cesumar de Ciência, Tecnologia e Inovação (ICETI), Coordenação de Aperfeiçamento de Pessoal de Nível Superior (CAPES) e Conselho Nacional de Desenvolvimento Científico e Tecnológico (CNPq) pela concessão de bolsas. 


\section{REFERÊNCIAS}

Al-Shaalan NH, Ali I, Alothman ZA, Al-Wahaibi LH, Alabdulmonem H. 2019. High performance removal and simulation studies of diuron pesticide in water on MWCNTs. Journal of Molecular Liquids, 289111039.

Armas EDd, Monteiro RTR, Antunes PM, Santos MAPFd, Camargo PBd, Abakerli RB. 2007. Diagnóstico espaço-temporal da ocorrência de herbicidas nas águas superficiais e sedimentos do Rio Corumbataí e principais afluentes. Química Nova, 30 1119-1127.

Brodie BC. 1859. On the Atomic Weight of Graphite. Philosophical Transactions of the Royal Society of London, 149 249-259.

Bumroongsakulsawat P, Khongthon W, Pavarajarn V. 2020. Degradation of diuron in water by electrochemical advanced oxidation in a microreactor: effects of anion contamination on degradation and toxicity. Journal of Environmental Chemical Engineering, 8 (4): 103824.

Cai W, Lai T, Dai W, Ye J. 2014. A facile approach to fabricate flexible all-solid-state supercapacitors based on MnFe2O4/ graphene hybrids. Journal of Power Sources, 255 (0): 170-178.

Chella S, Kollu P, Komarala EVPR, Doshi S, Saranya M, Felix S, . . Nirmala Grace A. 2015. Solvothermal synthesis of $\mathrm{MnFe} 2 \mathrm{O} 4$-graphene composite-Investigation of its adsorption and antimicrobial properties. Applied Surface Science, 327 (0): 27-36.

Chowdhury S, Balasubramanian R. 2014. Recent advances in the use of graphene-family nanoadsorbents for removal of toxic pollutants from wastewater. Advances in Colloid and Interface Science, 204 (0): 35-56.

de Souza FM, dos Santos OAA. 2018. Adsorption of Diuron from aqueous solution onto commercial organophilic clay: kinetic, equilibrium and thermodynamic study. Environ Technol 1-14.

Freundlich H. 1906. Adsorption in Solution. Phys. Chem. Soc., 408.

Ghaedi M, Ansari A, Habibi MH, Asghari AR. 2014. Removal of malachite green from aqueous solution by zinc oxide nanoparticle loaded on activated carbon: Kinetics and isotherm study. Journal of Industrial and Engineering Chemistry, 20 (1): $17-28$.

Guan C, Lv X, Han Z, Chen C, Xu Z, Liu Q. 2020. The adsorption enhancement of graphene for fluorine and chlorine from water. Applied Surface Science, 516146157.

Hummers WS, Offeman RE. 1958. Preparation of Graphitic Oxide. Journal of the American Chemical Society, 80 (6): 1339-1339.

Ji Y, Liu Q, Cheng M, Lai L, Li Z, Peng Y, Yang Y. 2013. Facile synthesis of reduced graphene oxide nanosheets by a sodium diphenylamine sulfonate reduction process and its electrochemical property. Materials Science and Engineering: C, 33 (7): 3811-3816.

Kovtyukhova NI, Ollivier PJ, Martin BR, Mallouk TE, Chizhik SA, Buzaneva EV, Gorchinskiy AD. 1999. Layer-byLayer Assembly of Ultrathin Composite Films from Micron-Sized Graphite Oxide Sheets and Polycations. Chemistry of Materials, 11 (3): 771-778. 
Kumar S, Nair RR, Pillai PB, Gupta SN, Iyengar MAR, Sood AK. 2014. Graphene Oxide-MnFe2O4 Magnetic Nanohybrids for Efficient Removal of Lead and Arsenic from Water. ACS Applied Materials \& Interfaces, 6 (20): 17426-17436.

Langmuir I. 1918. The adsorption og gases on plane suerfaces of glass, mica and platinium. J. Am. Chem. Soc., 408.

Lingamdinne LP, Koduru JR, Karri RR. 2019. A comprehensive review of applications of magnetic graphene oxide based nanocomposites for sustainable water purification. Journal of Environmental Management, 231 622-634.

Martín A, Escarpa A. 2014. Graphene: The cutting-edge interaction between chemistry and electrochemistry. TrAC Trends in Analytical Chemistry, 56 (0): 13-26.

Neolaka YAB, Lawa Y, Naat JN, Riwu AAP, Iqbal M, Darmokoesoemo H, Kusuma HS. 2020. The adsorption of $\operatorname{Cr}(\mathrm{VI})$ from water samples using graphene oxide-magnetic (GO-Fe3O4) synthesized from natural cellulose-based graphite (kusambi wood or Schleichera oleosa): Study of kinetics, isotherms and thermodynamics. Journal of Materials Research and Technology, 9 (3): 6544-6556.

Oller I, Gernjak W, Maldonado MI, Pérez-Estrada LA, Sánchez-Pérez JA, Malato S. 2006. Solar photocatalytic degradation of some hazardous water-soluble pesticides at pilot-plant scale. Journal of Hazardous Materials, 138 (3): 507-517.

Paz JEM, Garnica AIC, Curbelo FDS. 2018. ESTUDO DA ADSORÇÃO DE CHUMBO UTILIZANDO COMO ADSORVENTE BAGAÇO DE CANA-DE-AÇÚCAR ATIVADO. HOLOS, 08 (32): 16.

Pu S, Xue S, Yang Z, Hou Y, Zhu R, Chu W. 2018. In situ co-precipitation preparation of a superparamagnetic graphene oxide/Fe3O4 nanocomposite as an adsorbent for wastewater purification: synthesis, characterization, kinetics, and isotherm studies. Environmental Science and Pollution Research, 25 (18): 17310-17320.

Rawtani D, Khatri N, Tyagi S, Pandey G. 2018. Nanotechnology-based recent approaches for sensing and remediation of pesticides. Journal of Environmental Management, 206 749-762.

Ruiz FJ, Ripoll L, Hidalgo M, Canals A. 2019. Dispersive micro solid-phase extraction (D $\mu$ SPE) with graphene oxide as adsorbent for sensitive elemental analysis of aqueous samples by laser induced breakdown spectroscopy (LIBS). Talanta, 191 162-170.

Ruthven DM. 1984. Principles of Adsorption and Adsorption Processes, Ed.^Eds., New York: JOHN WILEY \& SONS, p.

Salvestrini S, Di Cerbo P, Capasso S. 2002. Kinetics of the chemical degradation of diuron. Chemosphere, 48 (1): 69-73.

Singh V, Joung D, Zhai L, Das S, Khondaker SI, Seal S. 2011. Graphene based materials: Past, present and future. Progress in Materials Science, 56 (8): 1178-1271.

Staudenmaier L. 1898. Verfahren zur Darstellung der Graphitsäure. Berichte der deutschen chemischen Gesellschaft, 31 (2): 1481-1487.

Tasmia, Shah J, Jan MR. 2020. Eco-friendly alginate encapsulated magnetic graphene oxide beads for solid phase microextraction of endocrine disrupting compounds from water samples. Ecotoxicology and Environmental Safety, 190110099.

Wang C, Feng C, Gao Y, Ma X, Wu Q, Wang Z. 2011. Preparation of a graphene-based magnetic nanocomposite for the removal of an organic dye from aqueous solution. Chemical Engineering Journal, 173 (1): 92-97. 
Yamaguchi NU, Bergamasco R, Hamoudi S. 2016. Magnetic MnFe2O4-graphene hybrid composite for efficient removal of glyphosate from water. Chemical Engineering Journal, 295 391-402.

Zbair M, El Hadrami A, Bellarbi A, Monkade M, Zradba A, Brahmi R. 2020. Herbicide diuron removal from aqueous solution by bottom ash: Kinetics, isotherm, and thermodynamic adsorption studies. Journal of Environmental Chemical Engineering, 8 (2): 103667.

Zhang Y, Chen B, Zhang L, Huang J, Chen F, Yang Z, . . Z Zhang Z. 2011. Controlled assembly of Fe3O4 magnetic nanoparticles on graphene oxide. Nanoscale, 3 (4): 1446-1450. 